\title{
THE JACOBSON RADICAL OF A CSL ALGEBRA
}

\author{
KENNETH R. DAVIDSON AND JOHN LINDSAY ORR
}

\begin{abstract}
Extrapolating from Ringrose's characterization of the Jacobson radical of a nest algebra, Hopenwasser conjectured that the radical of a CSL algebra coincides with the Ringrose ideal (the closure of the union of zero diagonal elements with respect to finite sublattices). A general interpolation theorem is proved that reduces this conjecture for completely distributive lattices to a strictly combinatorial problem. This problem is solved for all width two lattices (with no restriction of complete distributivity), verifying the conjecture in this case.
\end{abstract}

In [9] (cf. [3, Chapter 6]), Ringrose characterizes the Jacobson radical of a nest algebra. In [6], Hopenwasser described the appropriate generalization for reflexive algebras with commutative subspace lattice (CSL algebras), and verified his conjecture in a few special cases. The analogy with the nest case was pushed further in [8], clarifying the role of the carrier space. In this paper, we verify this conjecture for all width two CSL algebras. Moreover, we establish a framework for attacking the general problem and make significant progress in this direction.

Recall that the Jacobson radical of a Banach algebra is the intersection of the kernels of all irreducible continuous representations. It also coincides with those elements $T$ such that $A T$ is quasinilpotent for every $A$ in the algebra. Thus when $\mathscr{F}$ is a finite CSL, $\operatorname{Alg}(\mathscr{F})$ is a finite matrix algebra with certain coefficients running over $\mathscr{B}(\mathscr{H})$ (and the rest 0 ). There is a unique contractive projection $\Delta_{\mathscr{F}}$ of the algebra onto the diagonal $\mathscr{F} \prime \prime=\operatorname{Alg}(\mathscr{F}) \cap \operatorname{Alg}(\mathscr{F})^{*}$ given by $\Delta_{\mathscr{F}}(T)=\sum_{E} E T E$ as $E$ runs over the atoms of $\mathscr{F}$. It is easy to see that the radical of $\operatorname{Alg}(\mathscr{F})$ is precisely the set of zero diagonal elements ( $T$ such that $\left.\Delta_{\mathscr{F}}(T)=0\right)$, which we denote $\operatorname{Alg}_{0}(\mathscr{F})$. Indeed, this is a nilpotent ideal of order at most $|\mathscr{F}|$. Furthermore, it is easy to verify that $\operatorname{Alg}_{0}(\mathscr{F}) \cap \operatorname{Alg}(\mathscr{L})$ is an ideal in $\operatorname{Alg}(\mathscr{L})$ for all finite sublattices $\mathscr{F}$ of $\mathscr{L}$; and indeed, it is a nilpotent ideal. Thus it is contained in the radical. It follows that the norm closure

$$
\mathscr{R}(\mathscr{L}):=\overline{\bigcup_{\mathscr{F} \subset \mathscr{L}} \operatorname{Alg}_{0}(\mathscr{F}) \cap \operatorname{Alg}(\mathscr{L})}
$$

as $\mathscr{F}$ runs over all finite sublattices of $\mathscr{L}$ is a closed ideal of $\operatorname{Alg}(\mathscr{L})$ contained

Received by the editors November 16, 1992 and, in revised form, November 10, 1993.

1991 Mathematics Subject Classification. Primary 47D25; Secondary 16N20, 47A15, 05B40, 06A07, 05C05, 15A39, 47A57.

The first author was partially supported by an NSERC grant. The second author was partially supported by an NSERC International Fellowship and the National Science Foundation. 
in the Jacobson radical. In the case of nest algebras, Ringrose showed that this coincides with the radical. Hence we call $\mathscr{R}(\mathscr{L})$ the Ringrose ideal of $\operatorname{Alg}(\mathscr{L})$. Hopenwasser conjectures that the Ringrose ideal is equal to the radical for all CSL algebras.

Since $T \in \operatorname{Alg}_{0}(\mathscr{F})$ precisely when $\Delta \mathscr{F}(T)=0$, it is easy to see that $T$ belongs to $\mathscr{R}(\mathscr{L})$ precisely when, for all $\epsilon>0$, there is a finite sublattice $\mathscr{F}$ of $\mathscr{L}$ such that $\left\|\Delta_{\mathscr{F}}(T)\right\|<\epsilon$. This is known as the Ringrose condition.

There is a third, more technical, description of the ideal $\mathscr{R}(\mathscr{L})$ in terms of the carrier space. This is the compact Hausdorff space $\operatorname{Hom}(\mathscr{L}, 2)$ endowed with the order topology [6]. This is canonically homeomorphic to the maximal ideal space of $C^{*}(\mathscr{L})$, the $C^{*}$-algebra generated by the projections onto the subspaces of $\mathscr{L}$ [1]. For $\varphi \in \operatorname{Hom}(\mathscr{L}, 2)$, the set $\mathscr{E}_{\varphi}$ of test intervals for $\varphi$ consists of all intervals $E=L-M$ of $\mathscr{L}$ such that $M<L, \varphi(L)=1$ and $\varphi(M)=0$. This collection is an ultrafilter $\mathscr{U}_{\varphi}$ in the set of intervals of $\mathscr{L}$, and this pairing provides a natural bijection between $\operatorname{Hom}(\mathscr{L}, 2)$ and the space of all such ultrafilters. To $\varphi$, we associate an ideal $\mathscr{I}_{\varphi}$ consisting of all $T \in \operatorname{Alg}(\mathscr{L})$ such that

$$
\|T\|_{\varphi}:=\lim _{E \in \mathscr{Q}_{\varphi}}\|E T E\|=0
$$

Equivalently, this is the set of $T$ that for all $\epsilon>0$, there is a test interval $E \in \mathscr{U}_{\varphi}$ so that $\|E T E\|<\epsilon$.

The ultrafilter $\mathscr{U}_{\varphi}$ is principal if and only if it consists of all intervals containing an atom $A$. This corresponds to the seminorm $\|T\|_{\varphi}=\|A T A\|$. When this is nonzero, it is elementary to verify that $T$ is not in the radical. So we need only consider $T$ for which the diagonal part is zero.

The carrier space arises naturally in this context. For if $\pi$ is an irreducible representation of $\operatorname{Alg}(\mathscr{L})$, it is easy to see that $\pi(L)$ is invariant for $\pi(\operatorname{Alg}(\mathscr{L})$ ) for every $L \in \mathscr{L}$. Hence $\pi(L)$ is either 0 or $I$. Thus the restriction of $\pi$ to $\mathscr{L}$ is an element $\varphi$ of $\operatorname{Hom}(\mathscr{L}, 2)$. Notice that $\pi(E)=I$ for every $E \in \mathscr{E}_{\varphi}$. Therefore, since $\pi$ is continuous, one obtains

$$
\|\pi(T)\| \leq\|\pi\|\|T\|_{\varphi} .
$$

A fortiori, the ideal $\mathscr{I}_{\varphi}$ is contained in $\operatorname{ker} \pi$. Consequently, $\operatorname{rad}(\operatorname{Alg}(\mathscr{L}))$ contains the intersection of all the $\mathscr{I}_{\varphi}$.

It is clear that if $T$ satisfies the Ringrose condition, then $\|T\|_{\varphi}=0$ for all $\varphi$. The converse is a simple compactness argument. Thus the third equivalent characterization of the Ringrose ideal is

$$
\mathscr{R}(\mathscr{L})=\bigcap_{\varphi \in \operatorname{Hom}(\mathscr{L}, 2)} \mathscr{I}_{\varphi} .
$$

The arguments of Ringrose start with an operator $T$ in a nest algebra and a homomorphism $\varphi$ such that $\|T\|_{\varphi}>0$, and construct another operator $A$ so that $A T$ is not quasinilpotent. However, as Hopenwasser and Larson show, the order structure in $\mathscr{U}_{\varphi}$ is much more complicated for arbitrary commutative subspace lattices. This makes the construction of the operator $A$ much more difficult. The crux of our arguments is a combinatorial cum geometric analysis of the relationship between the intervals on which $T$ is large. We obtain the appropriate setting for constructing an operator $A$ so that $A T$ is not 
quasinilpotent in an arbitrary CSL algebra. However, in order to establish the existence of such a configuration, we have had to rely on certain planar geometric arguments. So our conclusive results hold only for width two lattices. For completely distributive lattices, the problem is reduced to a problem in finite combinatorics.

In this paper, all Hilbert spaces are separable. The algebra of all operators on $\mathscr{H}$ is denoted $\mathscr{B}(\mathscr{H})$. A CSL $\mathscr{L}$ is a complete lattice of commuting orthogonal projections (or the lattice of subspaces corresponding to their ranges). $\operatorname{Alg}(\mathscr{L})$ consists of all operators leaving each element of $\mathscr{L}$ invariant. An interval is the difference of two comparable elements of $\mathscr{L}$. An atom of $\mathscr{L}$ is a minimal interval. There is a useful partial order on the intervals given by $E \succ F$ provided that $F \operatorname{Alg}(\mathscr{L}) E=F \mathscr{B}(\mathscr{H}) E$. Also we say that $E$ and $F$ are completely noncomparable provided that $F \operatorname{Alg}(\mathscr{L}) E=E \operatorname{Alg}(\mathscr{L}) F=0$. An interval is said to be indecomposable if it is not the sum of two completely noncomparable intervals.

The lattice is endowed with its order topology, making it into a complete metrizable space. This topology is equivalent to the strong operator topology. The projections of $\mathscr{L}$ lie in a maximal abelian von Neumann algebra isomorphic to $L^{\infty}(\mu)$ for some measure $\mu$. The $L^{1}(\mu)$ metric $d_{\mu}$ is equivalent to this topology. We will write $\mu(L)$ for the $L^{1}(\mu)$ norm of the corresponding characteristic function, and $d_{\mu}(L, M)$ for the distance between $L$ and $M$ in the $L^{1}(\mu)$ norm.

A CSL $\mathscr{L}$ is called completely distributive provided that it satisfies a certain infinite distributive law. This property is equivalent to many nice approximation properties (cf. [3, Chapter 23]). It is fair to say that this is the largest tractable class of CSL's for most purposes. In particular, $\mathscr{L}$ is compact in this case [10]. In view of Lemma 2.2 below, we expect that the completely distributive case may be easier than the general conjecture.

A CSL $\mathscr{L}$ is finite width (specifically, width $n$ ) if it can be generated as a complete lattice by finitely many $(n)$ commuting chains of projections. This includes completely distributive lattices modeled on the $n$-cube such as the tensor product of $n$ commuting nests. But a nonatomic Boolean algebra of commuting projections is also width two. Arveson [2] introduced this class, and showed that they are all synthetic, a property that is not exploited here. For us, the usefulness of width two lattices is based on the fact that they are modeled on the square, and thus are amenable to planar geometric arguments.

The first author would like to thank Bernard Chevreau and the Universite de Bordeaux I for their hospitality while the work on this paper was completed.

Added in Proof. Ross Willard has recently shown us an ingenious counterexample to our Disjointness Conjecture (Conjecture 3.4 below). This provides a family of finite CSL's $\mathscr{L}_{m}$ each containing collections of intervals which cannot be cut by any $m$ lattice projections but such that, nevertheless, any pairwise orthogonal collection of intervals can be cut by a single projection. If we form the direct sum of these lattices, the Disjointness Conjecture fails for a totally atomic, hence completely distributive, lattice. We should mention however that the width of the lattices $\mathscr{L}_{m}$ grows rapidly with $m$. Thus the conjecture is still open for, in particular, finite width lattices (of width greater than two). Moreover, Theorem 3.9 guarantees that the Ringrose ideal is the Jacobson radical in any CSL for which the Disjointness Conjecture does hold. 


\section{UNIFORMITY OF THE RADICAL}

In this section, we show that the Jacobson radical of a CSL algebra has a strong uniform property. We also note that the Hopenwasser conjecture implies an even stronger version. First, a simple lemma.

Lemma 1.1. Suppose that $Y$ belongs to a CSL algebra $\mathscr{A}=\operatorname{Alg}(\mathscr{L})$, and that 1 belongs to $\sigma(Y)$. Then there exists a homomorphism $\varphi \in \operatorname{Hom}(\mathscr{L}, 2)$ so that for every test interval $E \in \mathscr{E}_{\varphi}, 1$ belongs to $\sigma\left(\left.E Y\right|_{E \mathscr{L}}\right)$.

Proof. This is a straightforward compactness argument. If the lemma were false, then for each $\varphi \in \operatorname{Hom}(\mathscr{L}, 2)$, there would be an interval $E_{\varphi} \in \mathscr{E}_{\varphi}$ such that $1 \notin \sigma\left(\left.E_{\varphi} Y\right|_{E_{\varphi} \mathscr{C}}\right)$. The set

$$
\mathscr{O}_{\varphi}=\left\{\psi \mid \psi\left(E_{\varphi}\right)=1\right\}
$$

is an open neighbourhood of $\varphi$. Thus these sets form an open cover of $\operatorname{Hom}(\mathscr{L}, 2)$, which is compact. A finite subcover yields interval projections $E_{i}$ so that $1 \notin \sigma\left(\left.E_{i} Y\right|_{E_{i} \mathscr{L}}\right)$ for $1 \leq i \leq n$ and $\sum_{i} E_{i} \geq I$. By replacing each $E_{i}$ by a possibly smaller interval projection, we may suppose that $\sum_{i} E_{i}=I$. It is routine to reorder such a family of interval projections so that $\sum_{1 \leq i \leq k} E_{i}$ belongs to $\mathscr{L}$ for every $k$. Thus it follows that $Y$ has an upper triangular form with respect to this (finite) decomposition, and that 1 is not in the spectrum of any of the diagonal entries. Hence $Y-I$ is invertible, contrary to hypothesis.

The following theorem strengthens the radical property, and enables some uniform estimations.

Theorem 1.2. Suppose that $\mathscr{A}=\operatorname{Alg}(\mathscr{L})$ is a CSL algebra, and that $X$ belongs to $\operatorname{rad}(\operatorname{Alg}(\mathscr{L}))$. Then,

$$
\lim _{n \rightarrow \infty} \sup _{A \in \text { ball }(\mathscr{A})}\left\|(A X)^{n}\right\|^{1 / n}=0 .
$$

Proof. Suppose that the theorem fails. Then there is a bounded sequence $A_{n} \in$ $\mathscr{A}$ so that $\left\|\left(A_{n} X\right)^{n}\right\|>1$. Let $X^{(\infty)}$ denote the operator acting on the space $\mathscr{H}^{(\infty)}$ consisting of the $l^{2}$ sum of countably many copies of $\mathscr{H}$ given by the diagonal operator with $X$ acting on each coordinate. This is an element of the CSL algebra $\mathscr{B}=\operatorname{Alg}\left(\mathscr{L}^{(\infty)}\right)$. Also, let $B=\sum_{n} \oplus A_{n}$ be defined analogously in $\mathscr{B}$. Then the spectral radius of $B X^{(\infty)}$ is at least 1 by construction. After replacing $B$ and each $A_{n}$ by a fixed scalar multiple, we may suppose that $1 \in \sigma\left(B X^{(\infty)}\right)$.

By Lemma 1.1, there is a homomorphism $\varphi \in \operatorname{Hom}(\mathscr{L}, 2)$ such that 1 belongs to $\sigma\left(\left.E^{(\infty)} B X^{(\infty)}\right|_{E^{(\infty)} \mathscr{H}(\infty)}\right)$ for every $E \in \mathscr{E}_{\varphi}$. In particular, for each such $E$ and integer $k$,

$$
1 \leq\left\|\left(E^{(\infty)} B X^{(\infty)} E^{(\infty)}\right)^{k}\right\|=\sup _{n}\left\|\left(E A_{n} X E\right)^{k}\right\| .
$$

Thus given $E$ and $k$, one may choose an integer $n(k)$ so that

$$
\left\|\left(E A_{n(k)} X E\right)^{k}\right\|>1-\frac{1}{k} .
$$


Note that $\varphi$ cannot be evaluation at an atom because the compression of $X$ to every atom is 0 . Hence, because $\mathscr{E}_{\varphi}$ is a nonprincipal ultrafilter and the norm is lower semicontinuous with respect to the weak-* topology, for an element $F$ of $\mathscr{E}_{\varphi}$ sufficiently small, one still has

$$
\left\|\left((E-F) A_{n(k)} X(E-F)\right)^{k}\right\|>1-\frac{1}{k} .
$$

Since $\mathscr{E}_{\varphi}$ is nonprincipal, it must contain a decreasing sequence $E_{n}$ with strong limit 0 . Choose lattice projections $L_{n}$ and $M_{n}$ so that $E_{n}=L_{n}-M_{n}$. To arrange things so that $L_{n}$ decreases and $M_{n}$ increases, recursively redefine these projections by replacing them by

$$
L_{n+1}^{\prime}=L_{n}^{\prime}\left(L_{n+1} \vee M_{n}^{\prime}\right)=E_{n} L_{n+1}+M_{n}^{\prime}
$$

and

$$
M_{n+1}^{\prime}=L_{n}^{\prime}\left(M_{n+1} \vee M_{n}^{\prime}\right)=E_{n} M_{n+1}+M_{n}^{\prime} .
$$

Let $L=\mathrm{s}-\lim _{n} L_{n}^{\prime}=\mathrm{s}-\lim _{n} M_{n}^{\prime}$. If $L$ does not belong to $E_{\varphi}$, then $F_{n}=L_{n}^{\prime}-L$ is a sequence of intervals in $\mathscr{E}_{\varphi}$ decreasing to 0 so that $F_{n}-F_{m}=L_{n}^{\prime}-L_{m}^{\prime}$ is always an interval. Otherwise, if $L$ does belong to $\mathscr{E}_{\varphi}$, then $F_{n}=L-M_{n}^{\prime}$ is such a sequence.

Thus it is an easy matter to construct a sequence $n(k)$ of integers and a sequence $G_{k}$ of pairwise orthogonal interval projections (of the form $F_{n}-F_{m}$ ) so that

$$
\left\|\left(G_{k} A_{n(k)} X G_{k}\right)^{k}\right\|>1-\frac{1}{k} .
$$

Define $A=\sum_{k \geq 1} G_{k} A_{n(k)} G_{k}$. Then

$$
\left\|(A X)^{k}\right\| \geq\left\|G_{k}(A X)^{k} G_{k}\right\|=\left\|\left(G_{k} A_{n(k)} X G_{k}\right)^{k}\right\|>1-\frac{1}{k} .
$$

This shows that $X$ is not in the radical of $\mathscr{A}$, contrary to hypothesis.

The following corollary will be very important in the sequel.

Corollary 1.3. Suppose that $X$ belongs to $\operatorname{rad}(\operatorname{Alg}(\mathscr{L}))$ and that $E_{i}$ is a family of pairwise orthogonal interval projections. Then the operator

$$
Y=\sum_{i \geq 1} E_{i} X E_{i}
$$

also belongs to $\operatorname{rad}(\operatorname{Alg}(\mathscr{L}))$.

Proof. Set $E=\sum_{i \geq 1} E_{i}$. Since $\operatorname{rad}(\operatorname{Alg}(\mathscr{L}))$ is an ideal, $Z=E X E$ belongs to the radical. Thus by Theorem 1.2 , for each $\epsilon>0$, there is an integer $N(\epsilon)$ so that for all $n \geq N(\epsilon)$ and $A \in \mathscr{A}$ with $\|A\| \leq 1$, one has $\left\|(A Z)^{n}\right\|<\epsilon^{n}$. Now set

$$
Y_{k}=\sum_{1 \leq i \leq k} E_{i} X E_{i}=\sum_{1 \leq i \leq k} E_{i} Z E_{i}
$$

It is evident that each $Y_{k}$ belongs to the radical. Our aim is to show that the estimates of the previous theorem can be done uniformly independent of $k$.

To this end, define

$$
U_{k}(\lambda)=\sum_{1 \leq i \leq k} \lambda_{i} E_{i}
$$


for $\lambda=\left(\lambda_{1}, \ldots, \lambda_{k}\right)$ in the $k$-torus $\mathbf{T}^{k}$. Let $d \lambda$ denote Haar measure on $\mathbf{T}^{k}$. Note that

$$
Y_{k}=\int_{\mathbf{T}^{k}} U_{k}(\lambda) Z U_{k}(\lambda)^{*} d \lambda
$$

Now fix an operator $A$ in the unit ball of $\mathscr{A}$. It will be shown that

$$
\left(A Y_{k}\right)^{n}=\int_{\mathbf{T}^{k}}\left(A U_{k}(\lambda) Z U_{k}(\lambda)^{*}\right)^{n} d \lambda
$$

Define $Z_{k}(\lambda)=U_{k}(\lambda) Z U_{k}(\lambda)^{*}$. Then

$$
\left(A Z_{k}(\lambda)\right)^{n}=\sum A E_{l_{1}} Z_{k}(\lambda) E_{l_{2}} A E_{l_{3}} Z_{k}(\lambda) E_{l_{4}} \cdots E_{l_{2 n-2}} A E_{l_{2 n-1}} Z_{k}(\lambda) E_{l_{2 n}},
$$

where the sum is taken over all choices of $l_{s}$ for $1 \leq s \leq 2 n$. However, because each $E_{i}$ is an interval, one has

$$
E_{i} A B C D \cdots X E_{i}=E_{i} A E_{i} B E_{i} C E_{i} D \cdots E_{i} X E_{i} .
$$

Thus one sees that a term $E_{i} A E_{l_{1}} B E_{l_{2}} \cdots E_{l_{p}} X E_{i}=0$ unless $i=l_{1}=\cdots=l_{p}$. Hence, in the sum above, one needs only consider the terms in which each projection $E_{i}$ occurs in a single, uninterrupted string.

Split the sum into two parts. The terms such that $l_{2 s-1}=l_{2 s}$ for $1 \leq$ $s \leq n$ are easily seen to sum to $\left(A Y_{k}\right)^{n}$. Every other term is a multiple of $\prod_{1 \leq s \leq n} \lambda_{l_{2 s-1}} \overline{\lambda_{l_{2 s}}}$ where, by the splitting, at least one of the terms $\lambda_{l_{2 s-1}} \overline{\lambda_{l_{2 s}}}$ is not 1 . Let $t$ denote the smallest $s$ for which this holds. Moreover, since the subscript $i=l_{2 t-1}$ occurs only in a single block, the term $\lambda_{i}=\lambda_{l_{2 t-1}}$ cannot be cancelled by any subsequent term in the product. It then follows that this term vanishes when one integrates over the torus $\mathbf{T}^{k}$. This establishes the desired formula.

The rest is easy. One obtains that for $n \geq N(\varepsilon)$,

$$
\begin{aligned}
\left\|\left(A Y_{k}\right)^{n}\right\| & \leq \int_{\mathbf{T}^{k}}\left\|\left(A Z_{k}(\lambda)\right)^{n}\right\| d \lambda \\
& =\int_{\mathbf{T}^{k}}\left\|\left(U_{k}(\lambda)^{*} A U_{k}(\lambda) Z\right)^{n}\right\| d \lambda \\
& <\varepsilon^{n} .
\end{aligned}
$$

The sequence $A Y_{k}$ converges to $A Y$ in the strong operator topology (and even in the strong-* topology). Multiplication is jointly continuous on the unit ball in the strong operator topology, and the norm is lower semicontinuous. Thus, for any integer $n \geq N(\varepsilon)$, one has

$$
\left\|(A Y)^{n}\right\|^{1 / n} \leq \limsup _{k \rightarrow \infty}\left\|\left(A Y_{k}\right)^{n}\right\|^{1 / n}<\varepsilon .
$$

Hence $Y$ belongs to the radical.

It is a well-known fact that if $\mathscr{A}$ is a Banach algebra with $\operatorname{radical} \operatorname{rad}(\mathscr{A})$, then the algebra $\mathscr{M}_{n}(\mathscr{A})$ of all $n \times n$ matrices with coefficients in $\mathscr{A}$ has radical $\mathscr{M}_{n}(\operatorname{rad}(\mathscr{A}))$. The main point is that $\mathscr{M}_{n}$ is simple and finite dimensional. The Hopenwasser conjecture suggests that for CSL algebras, the radical depends on the lattice structure, independent of multiplicity. In particular, it is easy to obtain: 
Lemma 1.4. If the Hopenwasser conjecture is valid, then $X \in \operatorname{rad}(\operatorname{Alg}(\mathscr{L}))$ implies that $X^{(\infty)}$ belongs to $\operatorname{rad}\left(\operatorname{Alg}\left(\mathscr{L}^{(\infty)}\right)\right)$.

This seems fairly innocuous, but as an easy corollary one obtains the following very strong radical condition.

Corollary 1.5. If the Hopenwasser conjecture is valid, then for every element $X$ in $\operatorname{rad}(\operatorname{Alg}(\mathscr{L}))$,

$$
\lim _{n \rightarrow \infty} \sup _{A_{i} \in \operatorname{ball}(\mathscr{A})}\left\|A_{1} X A_{2} X \cdots A_{n} X\right\|^{1 / n}=0 .
$$

Proof. Choose operators $A_{i, n}$ in the unit ball of $\mathscr{A}=\operatorname{Alg}(\mathscr{L})$ so that

$$
\left\|A_{1, n} X A_{2, n} X \cdots A_{n, n} X\right\| \geq \frac{1}{2} \sup _{A_{i} \in \operatorname{ball}(\mathscr{A})}\left\|A_{1} X A_{2} X \cdots A_{n} X\right\| .
$$

Define $A_{n}$ to be the $n+1 \times n+1$ matrix with $i, i+1$ entry equal to $A_{i, n}$ for $1 \leq i \leq n$, and all other coefficients equal to 0 . Set $A=\sum_{n \geq 1} \oplus A_{n}$. This is a weighted shift belonging to the unit ball of $\operatorname{Alg}\left(\mathscr{L}^{(\infty)}\right)$. Since $X \in \operatorname{rad}(\operatorname{Alg}(\mathscr{L}))$ implies that $X^{(\infty)}$ belongs to the radical of $\operatorname{Alg}\left(\mathscr{L}^{(\infty)}\right)$, one obtains

$$
\begin{aligned}
0 & =\lim _{n \rightarrow \infty}\left\|\left(A X^{(\infty)}\right)^{n}\right\|^{1 / n} \geq \lim _{n \rightarrow \infty}\left\|\left(A_{n} X^{(n+1)}\right)^{n}\right\|^{1 / n} \\
& =\lim _{n \rightarrow \infty}\left\|A_{1, n} X A_{2, n} X \cdots A_{n, n} X\right\|^{1 / n} \\
& =\lim _{n \rightarrow \infty} \sup _{A_{i} \in \operatorname{ball}(\mathscr{A})}\left\|A_{1} X A_{2} X \cdots A_{n} X\right\|^{1 / n} \cdot \square
\end{aligned}
$$

It is unknown for general Banach algebras whether the kernel of a topologically irreducible representation must contain the radical. However, it is clear in the case of a CSL algebra that, at least, the restriction of such a representation to the projections in $\mathscr{L}$ yields an element of $\operatorname{Hom}(\mathscr{L}, 2)$ because each $L \in \mathscr{L}$ is sent to an invariant projection, whence either 0 or $I$. Thus it follows easily that the kernel contains the Ringrose ideal $\mathscr{R}(\mathscr{L})$. So verification of the Hopenwasser conjecture would show that the kernel always contains the radical.

\section{FAMiLIES OF INTERVALS}

Suppose that an operator $T$ in $\operatorname{Alg}(\mathscr{L})$ fails to belong to the Ringrose ideal $\mathscr{R}(\mathscr{L})$. Thus there is some $\varphi$ in $\operatorname{Hom}(\mathscr{L}, 2)$ so that $\|T\|_{\varphi}>r>0$. Equivalently, any partition of the identity into finitely many orthogonal intervals of $\mathscr{L}$ has $\|E T E\|>r$ for at least one of the intervals. Thus we are led to consider the collection $\Omega(T)$ of all intervals $E$ of $\mathscr{L}$ for which $\|E T E\|>r$. This family is hereditary; that is, if $E \in \Omega(T)$ and $E<F$, then $F \in \Omega(T)$. This collection is also lower semicontinuous in the sense that whenever $E \in \Omega(T)$ and $E_{n}$ is a sequence of intervals increasing to $E$, then $E_{n}$ eventually belongs to $\Omega(T)$. Since this family is hereditary, this is equivalent to saying that $\Omega(T)$ is open in the $d_{\mu}$ metric on the intervals. Also, this collection is decomposable, meaning that if $E$ in $\Omega(T)$ is a countable sum of pairwise completely noncomparable intervals $E=\sum_{n \geq 1} E_{n}$, then there is an $n$ so that $E_{n}$ belongs to $\Omega(T)$. This is because $E T E \stackrel{=}{=} \sum_{n \geq 1} E_{n} T E_{n}$. Since $T$ fails the Ringrose condition, any finite partition into intervals has intervals in $\Omega(T)$. This is equivalent to saying that no finite collection of elements of $\mathscr{L}$ cuts every interval in $\Omega(T)$. (This is also a consequence of the fact that $\Omega$ contains the ultrafilter $\mathscr{U}_{\varphi}$. ) We say that 
a projection $L \in \mathscr{L}$ cuts an interval $E$ if $L E$ is a proper subinterval of $E$. In this section, we develop some general properties satisfied by such collections of intervals.

Definition 2.1. A lower semicontinuous, decomposable collection of intervals $\Omega$ is called hyporadical if no finite collection of lattice projections cuts them all. If, furthermore, for every positive integer $k$ there is a finite subcollection which cannot be cut by $k$ projections, then $\Omega$ is said to be radical.

If $\Omega$ is a collection of intervals in $\mathscr{L}$, we write $P(\Omega)$ to denote the smallest interval which dominates all the projections in $\Omega$. This exists since it is the meet of all intervals containing $\Omega$. It is important to obtain finite collections of intervals which cannot be cut by a given large number of projections in the lattice. Simple examples, e.g. the set of intervals $\{[0, t] \mid 0<t<1\}$ corresponding to the continuous nest in $L^{2}(0,1)$, have the property that no finite collection of projections in this nest cuts them all, but any finite subset can be cut by one projection. Of course, this family is not lower semicontinuous. A more serious example is given by the collection of all nonzero projections in the algebra $L^{\infty}(0,1)$ of all multiplication operators on $L^{2}(0,1)$. This collection is hyporadical. However, any finite subset can be cut by one projection. Indeed, the collection of all intervals corresponding to measurable sets with nonempty interior (with respect to the usual topology on $[0,1]$ ) can be cut with one projection. The problem is that this lattice is not completely distributive. It is also the case that the radical is zero for this algebra. Using complete distributivity, we can obtain the desired reduction.

Lemma 2.2. Every hyporadical collection $\Omega$ of intervals of a completely distributive CSL $\mathscr{L}$ is radical. Thus for any $k$, there is a finite collection of intervals in $\Omega$ which cannot be cut by any $k$ lattice projections. Moreover, these intervals may be taken to be indecomposable.

Proof. Since $\mathscr{L}$ is completely distributive, by Theorem 2.4 of [5], it has the property of semicontinuity. That is to say, for $L \in \mathscr{L}$ define

$$
\begin{aligned}
& L_{\delta}^{+}=\bigvee\left\{M \in \mathscr{L} \mid d_{\mu}(L, M)<\delta\right\}, \\
& L_{\delta}^{-}=\bigwedge\left\{M \in \mathscr{L} \mid d_{\mu}(L, M)<\delta\right\} .
\end{aligned}
$$

Then $L_{\delta}^{ \pm}$converge weak-* to $L$ as $\delta$ decreases to 0 . Thus, if $E=G-L$ is an interval in $\Omega$, then by approximating $G$ from below and $L$ from above in this way for sufficiently small $\delta$, we can find an interval $\widehat{E} \leq E$ in $\Omega$ such that any $L \in \mathscr{L}$ which cuts $\widehat{E}$ satisfies

$$
\min \left\{\mu(L E), \mu\left(L^{\perp} E\right)\right\} \geq \delta .
$$

Now suppose, contrary to our claim, that there is an integer $k$ so that every finite subset of $\Omega$ can be cut by $k$ lattice projections. This gives us a net in $\mathscr{L} \times \cdots \times \mathscr{L} \quad(k$ copies $)$ indexed by the finite subsets of $\Omega$. Since $\mathscr{L}$ is compact, our net has a cofinal subnet converging to, say, $\left(L_{1}, \ldots, L_{k}\right)$. Yet for any fixed $E \in \Omega$, this $k$-tuple is a strong limit of $k$-tuples each having one element which cuts $\widehat{E}$. Each such element splits $E$ into two pieces each of measure at least $\delta$. This property is preserved under strong limits. Hence one of $L_{1}, \ldots, L_{k}$ must cut each $E$ in $\Omega$, contradicting the hypothesis on $\Omega$. 
Since $\mathscr{L}$ is completely distributive, any interval $E$ can be decomposed into a countable direct sum of pairwise completely noncomparable intervals each of which is indecomposable. This is because the indecomposable intervals correspond to minimal projections in the largest orthocomplemented sublattice of $E \mathscr{L}$. Since this is a completely distributive Boolean lattice, it must be atomic. So each interval $E$ in $\Omega$ may be split into a countable sum of indecomposable intervals. Thus since hyporadical collections are decomposable, each $E \in \Omega$ may be replaced by an indecomposable subinterval.

Now we improve on this lemma to produce collections with disjoint supports requiring arbitrarily large numbers of cuts.

Lemma 2.3. Let $\Omega$ be a radical collection of intervals of a CSL $\mathscr{L}$. Then there is a sequence $\Omega_{k}$ of finite collections of intervals from $\Omega$ such that $\Omega_{k}$ cannot be cut by $k$ lattice projections and the projections $P\left(\Omega_{k}\right)$ are pairwise orthogonal. Proof. It suffices to show that for any natural number $k$ and radical collection $\Omega$, there is a finite collection of intervals $\Omega_{k}$ of $\Omega$ which cannot be cut by any $k$ lattice projections and a radical subcollection of intervals $\Omega^{\prime}$ such that $P\left(\Omega_{k}\right)$ and $P\left(\Omega^{\prime}\right)$ are orthogonal to each other. First, choose a finite collection $\Omega_{0}$ of intervals which cannot be cut by any $2 k+3$ lattice projections. Let $\mathscr{L}_{0}$ be a finite lattice of projections containing the members of $\Omega_{0}$ as intervals. Clearly, there is an atom $A$ of $\mathscr{L}_{0}$ so that the subset $\Omega^{\prime}$ of $\Omega$ consisting of all intervals $E$ in $\Omega$ dominated by $A$ is radical. Thus each interval $E_{0}$ of $\Omega_{0}$ is either orthogonal to $P\left(\Omega^{\prime}\right)$ or else $E_{0} \geq P\left(\Omega^{\prime}\right)$. Let $G$ and $L$ be projections in $\mathscr{L}_{0}$ so that $G-L=A$. Then $2 k+1$ projections in $\mathscr{L}$ cannot cut all the intervals $\Omega_{1}$ of $\Omega_{0}$ which are not cut by either $G$ or $L$. It is conceivable that $\Omega_{1}$ contains the interval $A$. The rest require more than $2 k$ cuts. These intervals split into two families, those dominated by $L$ and those orthogonal to $G$. One of these families requires more than $k$ projections to be cut. Let this be our definition of $\Omega_{k}$. Since either $L$ or $G^{\perp}$ dominates $P\left(\Omega_{k}\right)$, this is the desired decomposition. The proof is completed by a recursive use of this basic decomposition.

Suppose that $\Omega_{1}$ and $\Omega_{2}$ are two collections of intervals such that $\Omega_{1}$ cannot be cut by $p$ lattice projections, and for any $F \in \Omega_{1},\left\{E \in \Omega_{2} \mid E \leq F\right\}$ cannot be cut by any $k$ lattice projections. Then $\Omega_{2}$ cannot be cut by any $k+p$ lattice projections. This follows on supposing that $k+p$ lattice projections $L_{1}, \ldots, L_{k+p}$ can be chosen to cut all the intervals of $\Omega_{2}$. For each $F$ in $\Omega_{1}$, at least $k+1$ of these projections cut $F$. Thus one of $L_{1}, \ldots, L_{p}$ cuts $F$. This contradicts our hypothesis. There is a partial converse to this result which will be very useful throughout.

Lemma 2.4. Let $\Omega$ be a finite collection of intervals in a CSL which cannot be cut by any $f(k, p)=k p+k+p$ lattice projections. Let $\Omega^{*}$ be the set of those intervals $F$ which are minimal with respect to the property:

$$
\{E \in \Omega \mid E \leq F\} \text { cannot be cut by any } k \text { lattice projections. }
$$

Then $\Omega^{*}$ is a finite collection of intervals which cannot be cut by any $p$ lattice projections.

Proof. It is trivial to see that $\Omega^{*}$ is a finite set of intervals. Suppose, however, that $\Omega^{*}$ can be cut by $p$ lattice projections. These projections partition the 
space into at most $2^{p}$ intervals, none of which dominates a member of $\Omega^{*}$. Thus the intervals of $\Omega$ contained in each interval can be cut by $k$ projections. Hence, $2^{p} k+p$ projections suffice to cut the whole lot.

This number can be improved significantly. This is because the projections cutting a set of completely noncomparable intervals can be combined. The intervals of any CSL generated by $p$ projections can partitioned into at most $p+$ 1 groups of completely noncomparable intervals, namely those intervals which are the intersection of $j$ projections and $p-j$ complements. If $E_{1}, \ldots, E_{n}$ are completely noncomparable intervals and $P_{i}$ are projections which cut $E_{i}$, then the least element $P$ of $\mathscr{L}$ dominating all $E_{i} P_{i}$ satisfies $E_{i} P=E_{i} P_{i}$. Thus it suffices to use $f(k, p)=k(p+1)+p$ projections.

\section{LINKING MAPS}

In this section, we construct interpolating operators in $\operatorname{Alg}(\mathscr{L})$ needed to show that operators violating the radical condition are indeed not in the radical. The problem will be reduced to solving a system of inequalities. This system is dealt with by the following lemma.

Lemma 3.1. Let $A=\left(a_{i, j}\right)$ be a $q \times r$ matrix and $B=\left(b_{i, k}\right)$ be a $q \times s$ matrix with real coefficients. Then the linear programming problem

$$
\sum_{j=1}^{r} a_{i, j} \xi_{j} \leq \sum_{k=1}^{s} b_{i, k} \zeta_{k}, \quad \sum_{j=1}^{r} \xi_{j}=1, \quad \sum_{k=1}^{s} \zeta_{k}=1,
$$

has a solution for positive $\xi_{j}, \zeta_{k}$ if and only if there are no positive $\omega_{i}$ such that for all $j, k$,

$$
\sum_{i=1}^{q} b_{i, k} \omega_{i} \leq 1<\sum_{i=1}^{q} a_{i, j} \omega_{i} .
$$

Proof. Note first that if $T$ is an $m \times n$ matrix, then there is a nonzero $v \in \mathbf{R}^{n}$ such that $T v \geq 0$ (i.e. each entry of the vector $T v \in \mathbf{R}^{m}$ is nonnegative) if and only if $T^{t} \mathbf{R}_{+}^{m} \neq \mathbf{R}^{n}$. This is because $T v \geq 0$ if and only if

$$
0 \leq\langle T v, f\rangle=\left\langle v, T^{t} f\right\rangle \text { for all } f \in \mathbf{R}_{+}^{m} .
$$

By convexity theory this holds for some choice of $v$ if and only if the convex cone $T^{t} \mathbf{R}_{+}^{m}$ is not all of $\mathbf{R}^{n}$.

Now let $T$ be the $(q+r+s+2) \times(r+s)$ matrix:

$$
\left[\begin{array}{rr}
-A & B \\
I_{r} & 0 \\
0 & I_{s} \\
1_{r} & -1_{s} \\
-1_{r} & 1_{s}
\end{array}\right]
$$

(where $1_{k}$ is a $1 \times k$ row of 1 's). Then the linear programming problem of the statement is equivalent to solving $T v \geq 0$ for nonzero $v=(\xi, \zeta)$ in $\mathbf{R}^{r+s}$. Note that $T^{t} \mathbf{R}_{+}^{(q+r+s+2)}$ contains $\mathbf{R}_{+}^{(r+s)}$ and all real multiples of the column vector $\left(1_{r},-1_{s}\right)^{t}$. Thus, the linear programming problem has a solution if and only if the column vector $\left(-1_{r}, 0_{s}\right)^{t}$ is excluded from $T^{t} \mathbf{R}_{+}^{(q+r+s+2)}$. 
However $\left(-1_{r}, 0_{s}\right)^{t}$ belongs to $T^{t} \mathbf{R}_{+}^{(q+r+s+2)}$ if and only if

$$
-A^{t} x+y+u\left(1_{r}\right)^{t}=\left(-1_{r}\right)^{t}, \quad B^{t} x+z-u\left(1_{s}\right)^{t}=0
$$

has a solution for $x \in \mathbf{R}_{+}^{q}, y \in \mathbf{R}_{+}^{r}, z \in \mathbf{R}_{+}^{s}$ and $u \in \mathbf{R}$. This is equivalent to solving $A^{t} x \geq(u+1)\left(1_{r}\right)^{t}$ and $B^{t} x \leq u\left(1_{s}\right)^{t}$ for $x$ and $u$, which is clearly equivalent to the second condition in the statement.

Theorem 3.2. Let $\Omega_{1}=\left\{E_{i} \mid 1 \leq i \leq m\right\}$ and $\Omega_{2}=\left\{F_{j} \mid 1 \leq j \leq n\right\}$ be two families of disjoint intervals. Suppose that any element of $\mathscr{L}$ which meets every $F_{j} \in \Omega_{2}$ dominates some $E_{i}$ in $\Omega_{1}$. Then given unit vectors $\xi_{i}=E_{i} \xi_{i}$ and $\zeta_{j}=F_{j} \zeta_{j}$, there are nonnegative scalars $\alpha_{i}$ and $\beta_{j}$ and an operator $A$ in $\operatorname{Alg}(\mathscr{L})$ of norm 1 so that

$$
1=\sum_{1 \leq i \leq m} \alpha_{i}^{2}=\sum_{1 \leq j \leq n} \beta_{j}^{2}
$$

and

$$
A \sum_{1 \leq j \leq n} \beta_{j} \zeta_{j}=\sum_{1 \leq i \leq m} \alpha_{i} \xi_{i}
$$

Proof. It suffices to prove the result for all finite sublattices $\mathscr{F}$ of $\mathscr{L}$ which contain $\Omega_{1} \cup \Omega_{2}$ as a subset of its intervals. For then a standard compactness argument provides a solution. So let $\mathscr{F}$ be a finite sublattice of $\mathscr{L}$.

In [7], Hopenwasser solves the interpolation problem for single vectors. Namely, there is a contraction $A$ in $\operatorname{Alg}(\mathscr{L})$ satisfying $A \zeta=\xi$ if and only if

$$
\left\|L^{\perp} \xi\right\| \leq\left\|L^{\perp} \zeta\right\| \text { for all } L \in \mathscr{L} .
$$

From this, we deduce that a solution to our problem exists if and only if there is a nonnegative solution $\left\{u_{i}, v_{j}\right\}$ to the system of inequalities

$$
\begin{gathered}
\sum_{i}\left\|L^{\perp} \xi_{i}\right\|^{2} u_{i} \leq \sum_{j}\left\|L^{\perp} \zeta_{j}\right\|^{2} v_{j} \quad \text { for all } L \in \mathscr{F}, \\
\sum_{i} u_{i}=\sum_{j} v_{j}=1,
\end{gathered}
$$

where we have made the substitution $u_{i}=\alpha_{i}^{2}$ and $v_{j}=\beta_{j}^{2}$. By Lemma 3.1, there is no such solution exactly when there are nonnegative constants $\omega_{L}$ so that for all $i$ and $j$,

$$
\sum_{L \in \mathscr{F}}\left\|L^{\perp} \zeta_{j}\right\|^{2} \omega_{L} \leq 1<\sum_{L \in \mathscr{F}}\left\|L^{\perp} \xi_{i}\right\|^{2} \omega_{L}
$$

We shall show by induction that the system of inequalities (1) is never satisfied. We adopt as hypothesis that (1) cannot be solved in nonnegative $\omega_{L}$ when the $\zeta_{j}(j=1, \ldots, n)$ are any collection of vectors satisfying $\zeta_{j}=F_{j} \zeta_{j}$ and requiring exactly $s$ atoms of $\mathscr{F}$ to be supported. We proceed by induction on $s$. Since, in any event, $s \geq n$, we start with the case $s=n$.

Since the $F_{j}$ are pairwise orthogonal, in the case $s=n$ each $\zeta_{j}$ is supported on a single atom $A_{j} \leq F_{j}$. This case is particularly instructive to consider, since it motivates the general induction step to follow. Let $L_{0}$ be the smallest element of $\mathscr{F}$ dominating all the $A_{j}$. Then by the hypothesis on the families $\Omega_{1}$ and $\Omega_{2}$, there is an interval $E_{i_{0}}$ in $\Omega_{1}$ dominated by $L_{0}$. Hence $E_{i_{0}}$ 
decomposes as a sum of pairwise orthogonal intervals $E_{i_{0}}=\sum P_{j}$ such that each $A_{j}$ dominates $P_{j}$ in the ordering of intervals (i.e. $P_{j} \prec A_{j}$ ). We can then take $u_{i}=\delta_{i, i_{0}}$ and $v_{j}=\left\|P_{j} \xi_{i_{0}}\right\|^{2}$. This provides a solution to our original set of inequalities, and so, by Lemma 3.1, the system (1) has no solutions in this case.

Now suppose that the induction hypothesis holds for all values less than $s$, but suppose for a contradiction that there is a solution of the system (1) in case $s$. For each $j$, choose an atom $A_{j}$ of $\mathscr{F}$ so that $A_{j} \zeta_{j} \neq 0$. Again, let $L_{0}$ be the smallest element of $\mathscr{F}$ dominating all the $A_{j}$ and, by the hypothesis on the families $\Omega_{1}$ and $\Omega_{2}$, find an interval $E_{i_{0}}$ in $\Omega_{1}$ dominated by $L_{0}$. Then $E_{i_{0}}$ decomposes as a sum of atoms $E_{i_{0}}=\sum_{k} B_{k}$ so that every $B_{k}$ is dominated by some $A_{j}$ in the ordering of intervals. Then

$$
\sum_{k}\left\|B_{k} \xi_{i_{0}}\right\|^{2}=\left\|\xi_{i_{0}}\right\|^{2}=1<\sum_{L \in \mathscr{F}}\left\|L^{\perp} \xi_{i_{0}}\right\|^{2} \omega_{L}=\sum_{k}\left\|B_{k} \xi_{i_{0}}\right\|^{2} \sum_{L \perp B_{k}} \omega_{L} .
$$

Thus for some $k_{0}$, one has $\sum_{L \perp B_{k_{0}}} \omega_{L}>1$.

Extend the list $A_{1}, \ldots, A_{n}$ to the complete set of atoms $A_{1}, \ldots, A_{s}$ which support some $\zeta_{j}$. For each $j$, define $c_{j}=\sum_{L \perp A_{j}} \omega_{L}$. Then since there is an $A_{j_{0}} \succ B_{k_{0}}$, it follows that

$$
c_{j_{0}} \geq \sum_{L \perp B_{k_{0}}} \omega_{L}>1
$$

However,

$$
\sum_{1 \leq j \leq s}\left\|A_{j} \zeta_{j_{0}}\right\|^{2} c_{j}=\sum_{L \in \mathscr{F}}\left\|L^{\perp} \zeta_{j_{0}}\right\|^{2} \omega_{L} \leq 1
$$

Hence

$$
\sum_{j \neq j_{0}}\left\|A_{j} \zeta_{j_{0}}\right\|^{2} c_{j} \leq 1-\left\|A_{j_{0}} \zeta_{j_{0}}\right\|^{2} c_{j_{0}}<1-\left\|A_{j_{0}} \zeta_{j_{0}}\right\|^{2}=\left\|A_{j_{0}}^{\perp} \zeta_{j_{0}}\right\|^{2}
$$

In particular, this implies that $\left\|A_{j_{0}} \zeta_{j_{0}}\right\|<1$.

Replace $\zeta_{j_{0}}$ by $\zeta_{j_{0}}^{\prime}:=\left\|A_{j_{0}}^{\perp} \zeta_{j_{0}}\right\|^{-1} A_{j_{0}}^{\perp} \zeta_{j_{0}}$. This new set of $\zeta_{j}$ 's are supported on $s-1$ atoms. Moreover,

$$
\sum_{L \in \mathscr{F}}\left\|L^{\perp} \zeta_{j_{0}}^{\prime}\right\|^{2} \omega_{L}=\left\|A_{j_{0}}^{\perp} \zeta_{j_{0}}\right\|^{-2} \sum_{j \neq j_{0}}\left\|A_{j} \zeta_{j_{0}}\right\|^{2} c_{j}<1
$$

Hence equation (1) is still satisfied for this new set of vectors. This contradicts the induction hypothesis. So the theorem follows.

Remark 3.3. Note that the theorem above is still valid if the vectors $\zeta_{j}$ have norm greater than 1. For one can incorporate into $A$ a contraction which multiplies each $\zeta$ by an appropriate scalar to reduce them to norm 1 .

The main obstacle remaining is the disjointness of the intervals in a radical collection. In the next section, we resolve this problem for width two lattices. In general, we have been unable to accomplish this. We will formulate this as a conjecture, and then show that a positive resolution of this conjecture yields a solution to the radical conjecture at least in the completely distributive case.

Conjecture 3.4 (Disjointness). Let $\mathscr{L}$ be a CSL. Then there is a positive integer $M$ such that if $\Omega$ is a finite set of indecomposable intervals in $\mathscr{L}$ which cannot 
be simultaneously cut by $M$ lattice projections, then there is a subcollection $\Omega^{\prime}$ consisting of pairwise disjoint intervals which cannot be cut by a single lattice projection.

The need for indecomposable intervals is made evident by the following example. Consider the algebra $l^{\infty}$ acting on $l^{2}$. This is a CSL algebra with subspace lattice consisting of the atomic Boolean algebra of all diagonal projections. This lattice is also width two and completely distributive. Let $\Omega$ consist of all projections onto the sum of any two atoms. We claim that $\Omega$ is radical. Indeed, consider the subset $\Omega_{k}$ consisting of those projections supported on the first $2^{k}+1$ atoms. Any $k$ lattice projections divide this set of atoms into at most $2^{k}$ intervals. By the pigeonhole principle, at least one of these intervals has cardinality at least two. Thus $\Omega_{k}$ cannot be cut by $k$ lattice projections. On the other hand, any subcollection of pairwise orthogonal projections in $\Omega$ can easily be cut by a single projection!

To facilitate the description of our construction, we introduce the following notion. The usefulness of the concept has already been demonstrated in Theorem 3.2.

Definition 3.5. A bush of order 1 for a collection $\Omega$ of intervals is a finite subset $\mathscr{B}_{1}=\Psi_{1} \cup \dot{\cup} \Phi_{1}$ consisting of pairwise disjoint intervals in $\Omega$ divided into two disjoint subsets $\Psi_{1}$ and $\Phi_{1}$ such that any projection which meets each $F \in \Phi_{1}$ must dominate some $E \in \Psi_{1}$. A bush of order $n$ for $\Omega$ is a bush of order 1 $\mathscr{B}_{n}=\Psi_{n} \cup \Phi_{n}$ of pairwise disjoint intervals (not necessarily from $\Omega$ ) such that each interval in $\mathscr{B}_{n}$ dominates a bush of order $n-1$ for $\Omega$.

We start with a simple observation. In fact, an examination of the proof shows that the condition " $\{E \in \Omega \mid E \leq G\}$ cannot be cut by one projection" can be replaced by " $\{E \in \Omega \mid E \leq G\}$ contains at least two intervals". However, the present formulation suits our purposes for the induction argument to follow.

Lemma 3.6. If $\Omega^{*}$ is a finite collection of disjoint intervals which cannot be cut by one projection, and $\Omega$ is a collection of disjoint intervals such that for each $G \in \Omega^{*}$ the set $\{E \in \Omega \mid E \leq G\}$ cannot be cut by one projection, then $\Omega$ contains a bush of order 1 .

Proof. Choose $\Phi$ so that it contains one interval $F \in \Omega$ dominated by each $G \in \Omega^{*}$. Suppose that $L$ meets each $F \in \Phi$. Then $L$ meets each $G \in \Omega^{*}$. Since there must be some $G_{0} \in \Omega^{*}$ which is not cut by $L$, one must have $G_{0} \leq L$. Thus, $G_{0}$ and hence $L$ dominates an interval $F \in \Omega \backslash \Phi$.

Let us verify that, assuming our conjecture, bushes of high order belong to radical collections.

Lemma 3.7. Assuming the Disjointness Conjecture for $\mathscr{L}$, there are integers $M(k)$ so that if $\Omega$ is a finite collection of intervals requiring more than $M(k)$ cuts, then it contains a bush of order $k$.

Proof. Let $M(1)=f(M, M)$, where $M$ is the constant in the Disjointness Conjecture, and $f$ is the function of Lemma 2.4. If $\Omega$ is a finite collection which cannot be cut by $M(1)$ projections, Lemma 2.4 shows that the finite collection $\Omega^{*}$ of minimal intervals containing subcollections of $\Omega$ which require more than $M$ cuts itself requires more than $M$ cuts. Hence, $\Omega^{*}$ contains a disjoint subcollection $\mathscr{F}$ which cannot be cut by a single projection. Likewise, 
each element $F \in \mathscr{F}$ must dominate a disjoint collection of intervals in $\Omega$ which cannot be cut by one projection. Thus Lemma 3.6 applies.

Now recursively define $M(k)=f(M(1), M(k-1))$. Proceeding as above, let $\Omega^{*}$ be the collection of all minimal intervals $F$ such that the collection of intervals $E \in \Omega$ dominated by $F$ requires at least $M(k-1)$ cuts. Then by Lemma $2.4, \Omega^{*}$ requires more than $M(1)$ projections to be cut. From the previous paragraph, we see that $\Omega^{*}$ contains a bush $\mathscr{B}$ of order 1 . Each element $F \in \mathscr{B}$ dominates a bush of order $k-1$ for $\Omega$ by the induction hypothesis. Hence we obtain a bush $\mathscr{B}$ of order $k$.

Now let us construct the interpolating operators.

Lemma 3.8. Suppose that $\mathscr{B}$ is a bush of order $k$. Let $\beta_{1}(\mathscr{B})$ denote the collection of all the intervals in all the bushes of order 1 in the hierarchy of $\mathscr{B}$ (all the "highest branches"). Suppose that $\|E X E\|>1$ for all intervals $E$ in $\beta_{1}(\mathscr{B})$, and let $Y=\sum_{E \in \beta_{1}(\mathscr{B})} E X E$. Then there is an operator $A=P(\mathscr{B}) A P(\mathscr{B})$ in $\operatorname{Alg}(\mathscr{L})$ of norm 1 such that

$$
\left\|P(\mathscr{B}) Y(A Y)^{2^{k}-1} P(\mathscr{B})\right\|>1 .
$$

Proof. We proceed by induction on $k$. Take in the induction hypothesis the further stipulation that $A=P A Q$ for certain projections $P$ and $Q$ in $\mathscr{L}^{\prime \prime}$ dominated by $P(\mathscr{B})$, so that there is a unit vector $\xi \in P^{\perp} P(\mathscr{B}) \xi$ and a vector $\zeta=Q^{\perp} P(\mathscr{B}) \zeta$ of norm greater than 1 such that $Y(A Y)^{2^{k}-1} \xi=\zeta$. The case $k=0$ can be taken to be the statement that since $\|E X E\|>1$, there is a unit vector $\xi=E \xi$ and a vector $\zeta=E \zeta$ with $\|\zeta\|>1$ so that $Y \xi=E X \xi=\zeta$. So the hypothesis is valid for $k=0$.

Suppose that the induction hypothesis is true for all integers less than $k$. Write the bush as $\mathscr{B}=\Psi \dot{\cup} \Phi$. Since each interval $G \in \mathscr{B}$ dominates a bush of order $k-1$, the induction hypothesis provides projections $P_{G}$ and $Q_{G}$ less than $G$, vectors $\xi_{G}=P_{G}^{\perp} G \xi_{G}$ and $\zeta_{G}=Q_{G}^{\perp} G \zeta_{G}$ with $1=\left\|\xi_{G}\right\|<\left\|\zeta_{G}\right\|$, and an operator $A_{G}=P_{G} A_{G} Q_{G}$ of norm one in $\operatorname{Alg}(\mathscr{L})$ such that

$$
Y\left(A_{G} Y\right)^{2^{k-1}-1} \xi_{G}=\zeta_{G}
$$

Set $B=\sum_{G \in \mathscr{B}} A_{G}$, and note that this has norm 1 .

The collections $\Psi$ and $\Phi$ and vectors $\xi_{E}, E \in \Psi$, and $\zeta_{F}, F \in \Phi$, satisfy the hypotheses of Theorem 3.2 (see Remark 3.3). Thus there are nonnegative scalars $\alpha_{E}$ and $\beta_{F}$ such that

$$
\sum_{E \in \Psi} \alpha_{E}^{2}=\sum_{F \in \Phi} \beta_{F}^{2}=1,
$$

and an operator $T=P(\Psi) T P(\Phi)$ in $\operatorname{Alg}(\mathscr{L})$ of norm 1 such that

$$
T \sum_{F \in \Phi} \beta_{F} \zeta_{F}=\sum_{E \in \Psi} \alpha_{E} \xi_{E} .
$$

Since the $\zeta_{F}$ are supported on the intervals $F-Q_{F}$ and the vectors $\xi_{E}$ are supported on the intervals $E-P_{E}$, there is no loss in assuming that the domain and range of $T$ are contained in $\sum_{F \in \Phi} F-Q_{F}$ and $\sum_{E \in \Psi} E-P_{E}$ respectively. In particular, the domain and range of $T$ are orthogonal to the domain and range of $B$, respectively. Thus the operator $A=B+T$ belongs to $\operatorname{Alg}(\mathscr{L})$ and has norm 1. 
Now define

$$
\xi=\sum_{F \in \Phi} \beta_{F} \xi_{F} \quad \text { and } \quad \zeta=\sum_{E \in \Psi} \alpha_{E} \zeta_{E}
$$

It is evident that

$$
\begin{aligned}
Y(A Y)^{2^{k}-1} \xi & =Y(A Y)^{2^{k-1}-1} T Y(A Y)^{2^{k-1}-1} \xi \\
& =Y(A Y)^{2^{k-1}-1} T \sum_{F \in \Phi} \beta_{F} \zeta_{F} \\
& =Y(A Y)^{2^{k-1}-1} \sum_{E \in \Psi} \alpha_{E} \xi_{E}=\zeta .
\end{aligned}
$$

Moreover, the operator $A$ has the form $P A Q$, where

$$
P=P(\Psi) \vee \sum_{F \in \Phi} P_{F}
$$

is orthogonal to the support of $\xi$, and

$$
Q=P(\Phi) \vee \sum_{E \in \Psi} Q_{E}
$$

is orthogonal to the support of $\zeta$. Thus the induction hypothesis is established.

In the following theorem, complete distributivity is needed for two important points. The first is to show that hyporadical collections are radical. The second is to replace intervals by indecomposable subintervals. Both of these facts are contained in Lemma 2.2 .

Theorem 3.9. The Ringrose ideal coincides with the Jacobson radical of $\operatorname{Alg}(\mathscr{L})$ for every completely distributive CSL for which the Disjointness Conjecture holds. Proof. In view of the discussion in the Introduction, it must be shown that if $X$ is not in $\mathscr{R}(\mathscr{L})$, then $X$ is not in the radical. In this case, there is an $r>0$ so that $\Omega=\{E \mid\|E X E\|>r\}$ is hyporadical. After scaling, we may suppose that $r=1$. By Lemma 2.2, $\Omega$ is radical. Then by Lemma 2.3, there are finite subcollections $\Omega_{k}$ of indecomposable intervals such that $P\left(\Omega_{k}\right)$ are pairwise orthogonal, and $\Omega_{k}$ cannot be cut by $M(k)$ projections. By Lemma 3.7, $\Omega_{k}$ contains a bush $\mathscr{B}_{k}$ of order $k$.

By Corollary 1.3, $X$ belongs to the radical only if

$$
Y=\sum_{k \geq 1} \sum_{E \in \beta_{1}\left(B_{k}\right)} E X E
$$

does. Now by Lemma 3.8, there are operators $A_{k}=P\left(\Omega_{k}\right) A_{k} P\left(\Omega_{k}\right)$ in the ball of $\mathscr{A}$ so that

$$
\left\|P\left(\Omega_{k}\right) Y\left(A_{k} Y\right)^{2^{k}-1} P\left(\Omega_{k}\right)\right\|>1 .
$$

Thus if we set $A=\sum_{k \geq 1} A_{k}$, then $A$ is in the unit ball of $\mathscr{A}$ and $A Y$ is not quasinilpotent. Hence $\bar{Y}$, and also $X$, is not in the radical.

\section{WIDTH TWO LATTICES}

In this section, we verify the Disjointness Conjecture for width two lattices. We are able to do this without assuming complete distributivity. Hence we are able to verify the Hopenwasser conjecture for this class of lattices. 
It is easy to see that the tensor product of two copies of the Volterra nest on $L^{2}(0,1)$ is isomorphic to the lattice of decreasing Lebesgue measurable sets (modulo null sets) on the unit square. It is not so obvious that the nonatomic Boolean algebra of Lebesgue measurable subsets of $(0,1)$ can also be modeled as decreasing measurable subsets of the unit square if the measure is taken to be linear Lebesgue measure on the diagonal $x+y=1$. Indeed, all width two lattices can be modeled on subsets of the unit square. This is a special case of Arveson's representation theorem [2].

Lemma 4.1. Let $\mathscr{L}$ be a width two CSL. Then there is a Borel measure $\mu$ on the unit square $X$ such that the lattice $\mathscr{L}(X, \leq, \mu)$ of $\mu$-equivalence classes of decreasing sets of $X$ is lattice isomorphic to $\mathscr{L}$.

Proof. Let $\mathscr{N}$ and $\mathscr{M}$ be two nests generating $\mathscr{L}$. If $\xi$ is a unit separating vector for $\mathscr{L}$, then the maps from $t \in[0,1]$ to

$$
\bigwedge\{M \in \mathscr{M} \mid\|M \xi\| \geq t\} \text { and } \bigwedge\{N \in \mathscr{N} \mid\|N \xi\| \geq t\}
$$

give parametrizations of $\mathscr{M}$ and $\mathscr{N}$ respectively which are strongly continuous for increasing $t$. Thus $F(s, t)=\left\|\left(M_{s} \wedge N_{t}\right) \xi\right\|$ is an increasing map on the unit square and is continuous for increasing limits. We can define a LebesgueStieltjes measure $\mu$ on the unit square such that $\mu([0, s] \times[0, t])=F(s, t)$. It is straightforward to verify that the map which takes the projection corresponding to $[0, s] \times[0, t]$ in $\mathscr{L}(X, \leq, \mu)$ to the projection $M_{s} N_{t}$ in $\mathscr{L}$ is well defined, and extends to a lattice isomorphism between the lattices (finitely) generated by the projections corresponding to the $[0, s] \times[0, t]$ and the $M_{s} N_{t}$. Moreover because this map is measure-preserving, both it and its inverse are weak-* continuous and extend by continuity to lattice isomorphisms.

For each point $\xi$ in the unit square, we define the two closed sets $L(\xi)$ and $U(\xi)$ to be, respectively, the set of all $\zeta \leq \xi$ in the unit square and the set of all $\zeta \geq \xi$ relative to the product partial order on $\mathbf{R}^{2}$. By analogy with the definitions for lattices, we say that a subset $E$ of a partially ordered set is an interval if whenever $x \leq y$ belong to $E$, then every $x \leq z \leq y$ belongs to $E$. It is easy to see that every interval of $\mathscr{L}$ has a representative which is an interval set. Moreover, an indecomposable interval has a representative which is a connected interval set. In order to keep a notational consistency between sets and projections, we will write $L^{\perp}$ for the set complement of $L$. It is important for us to retain the connection with the measure $\mu$. So we say that a set $L$ corresponding to a projection cuts an interval $E$ if both $L \cap E$ and $L^{\perp} \cap E$ have positive $\mu$-measure. Similarly, we say that an interval $E$ contains $F$ if $\mu(F \backslash E)=0$.

Our first goal is to use the planar geometry to extend Lemma 2.2 to cover certain hyporadical collections $\Omega(X)$ in width two lattices which are not completely distributive. In view of the semicontinuity characterization of complete distributivity [5], let us say that a lattice is completely non-CD if

$$
\bigwedge_{\varepsilon>0} \bigvee_{\mu(L)<\varepsilon} L=I
$$

An interval $E$ of $\mathscr{L}$ is called completely non-CD if the lattice $E \mathscr{L}$ is completely non-CD. 


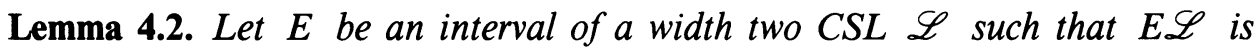
completely non-CD. Then $E \mathscr{L}$ is complemented.

Proof. Without loss of generality, $E=I$. Note that $\mathscr{L}$ is nonatomic. Model $\mathscr{L}$ on the square as in Lemma 4.1. The sublattice of $\mathscr{L}$ of all elements which are finite unions of the basic projections of the form $M_{s} \wedge N_{t}$ is dense in $\mathscr{L}$. Hence

$$
L_{\varepsilon}:=\left\{\xi=(s, t) \mid \mu\left(M_{s} \wedge N_{t}\right) \leq \varepsilon\right\}
$$

is a decreasing set in the square with $\mu\left(L_{\varepsilon}\right)=1$. In fact, $L_{\varepsilon}$ is closed. This follows from the fact that all horizontal and vertical lines have $\mu$-measure 0 . To see that this is the case, suppose that a horizontal line segment $H$ had positive measure. Then the restriction of $\mathscr{L}$ to $H$ is a nontrivial nest. This contradicts the completely non-CD property.

Moreover, $L_{\varepsilon}$ is an increasing function of $\varepsilon$. Thus $L_{0}=\bigcap_{\varepsilon>0} L_{\varepsilon}$ is also a closed decreasing set with $\mu\left(L_{0}\right)=1$. Let $S$ be the minimal boundary of $L_{0}$ given by

$$
S:=L_{0} \backslash \bigcup_{\xi \in L_{0}} L(\xi) \backslash\{\xi\} .
$$

This removes at most countably many horizontal and vertical line segments from the topological boundary of $L_{0}$. What remains is the graph of a left continuous decreasing function $f$. We claim that $\mu(S)=1$. The point is that $\mu(L(\xi))=0$ for every $\xi$ in $L_{0}$. Let $C$ be a countable dense subset of $L_{0}$ including the end points of line segments removed from the boundary. Then

$$
S=L_{0} \backslash\left(\bigcup_{\xi \in C} L(\xi) \backslash\{\xi\}\right) .
$$

Hence $\mu(S)=1$ because we have removed countably many sets of $\mu$-measure 0 .

It is easy to see that each contiguous segment $\{(s, f(s)) \mid a \leq s \leq b\}$ of the graph $S$ is an element of $\mathscr{L}$ corresponding to the rectangle $M_{b} \wedge N_{f(a)}$. Moreover, these elements generate $\mathscr{L}$ as a lattice. But it is also clear that these projections are complemented in $\mathscr{L}$. Hence $\mathscr{L}$ is a nonatomic complemented lattice.

It should be remarked that this lemma fails already in the width three case. A continuous nest tensored with the lattice of $L^{\infty}(0,1)$ is a counterexample.

Every $C^{*}$-algebra is semisimple. Hence when $\mathscr{L}$ is complemented, the radical of $\operatorname{Alg}(\mathscr{L})$ is 0 . Thus if $X$ is in the radical of $\operatorname{Alg}(\mathscr{L})$, it must satisfy $E X E=0$ for every interval $E$ such that $E \mathscr{L}$ is complemented. So by the lemma above, this means that in the width two case, we can restrict our attention to hyporadical collections which do not contain any completely non-CD intervals. Now any interval in the square is bounded by two decreasing curves. Thus, unless it lies on such a curve, it has (topological) interior. An interval consisting of a decreasing curve splits as the union of a completely non-CD piece and a $C D$ piece which is the union of horizontal and vertical line segments. Given a measure $\mu$, the square is easily seen to decompose into a countable union of decreasing curves supporting nonzero measure and the rest, which by itself is CD. Let $\mu_{0}$ denote this CD part of the measure. Let us say that $\mu_{0}$ has 
dense support relative to $\mu$ if for every open set $\mathscr{O}$ in the square, $\mu_{0}(\mathscr{O})=0$ only if $\mu(\mathscr{O})=0$. The next lemma is a stepping stone to the final result.

Lemma 4.3. Suppose that $\mu$ is a measure on the square such that the CD part $\mu_{0}$ has dense support relative to $\mu$. Suppose that $\Omega$ is a hyporadical collection that does not contain any completely non-CD intervals. Then $\Omega$ is radical.

Proof. Suppose, contrary to our claim, that there is an integer $k$ such that every finite subset of $\Omega$ can be cut by $k$ lattice projections. This gives us a net in $\mathscr{L} \times \cdots \times \mathscr{L}$ ( $k$ copies) indexed by the finite subsets of $\Omega$. Since the unit ball of $\mathscr{L}^{\prime \prime}$ is weak-* compact, our net has a cofinal subnet $\left(L_{1}^{\alpha}, \ldots, L_{k}^{\alpha}\right), \alpha \in \mathbf{A}$, converging to a $k$-tuple $\left(X_{1}, \ldots, X_{k}\right)$, where each $X$ is a positive contraction in $\mathscr{L}^{\prime \prime}$ such that the spectral projections $E_{X}\{[a, 1]\}$ belong to $\mathscr{L}$ for all real $a$. In particular, the projections $L_{i}^{+}=E_{X_{i}}\{1\}$ and $L_{i}^{-}=E_{X_{i}}\{(0,1]\}$ belong to $\mathscr{L}$.

Let $\Sigma$ be the complement in the square of the countably many decreasing curves supporting the non-CD part of $\mathscr{L}$. (Note that $\Sigma$ will contain all horizontal and vertical line segments of nonzero measure.) Then the restriction of the projections in the net to $\Sigma$ belong to a CD lattice $\mathscr{L}_{0}=E(\Sigma) \mathscr{L}$. Now the set of projections in $\mathscr{L}_{0}$ is compact in the strong (hence weak-*) topology. This means that $E(\Sigma) X_{i}$ are projections. Furthermore, $E(\Sigma) L_{i}^{-}=E(\Sigma) L_{i}^{+}$for each $i$. This means that the intervals $L_{i}^{+}-L_{i}^{-}$are completely non-CD.

The intervals $\left\{L_{1}^{ \pm}, \ldots, L_{n}^{ \pm}\right\}$chop the square into finitely many intervals. Thus one of these intervals, say $F$, dominates a hyporadical subcollection $\Omega_{1}$ of $\Omega$. Since $F$ cannot be completely non-CD, the comments of the previous paragraph show that $F L_{i}^{+}=F L_{i}^{-}=F X_{i} \in\{0, F\}$ for each $i$. Whenever a sequence of projections converges weak-* to a projection, it also converges in the strong topology. So $F L_{i}^{\alpha}$ converges strongly to the projection $F X_{i}$, which is either 0 or $F$. Hence $\mu\left(F L_{i}^{\alpha}\right)$ converges to either 0 or $\mu(F)$.

Let

$$
P=\bigwedge_{\varepsilon>0} \bigvee_{\mu(F L)<\varepsilon} F L \text { and } Q=\bigvee_{\varepsilon>0} \bigwedge_{\mu\left(F L^{\perp}\right)<\varepsilon} F L
$$

It is clear that $P$ and $Q$ are completely non-CD intervals. Thus after two more cuts, we can replace $F$ by $G=F-P-Q$. This still must support a hyporadical collection $\Omega_{2}$. Plus, it now follows that when $F L_{i}=0$, say for $i \in I$,

$$
\bigwedge_{\alpha \in \mathbf{A} \beta>\alpha} \bigvee_{\beta} G L_{i}^{\beta}=0
$$

Similarly, when $F L_{i}=F$ for $i \in J=\{1, \ldots, k\} \backslash I$, one obtains

$$
\bigvee_{\alpha \in A} \bigwedge_{\beta>\alpha} G L_{i}^{\beta}=G \text {. }
$$

This now leads to a contradiction because the increasing net

$$
E_{\alpha}=\bigwedge_{i \in J} \bigwedge_{\beta>\alpha} G L_{i}^{\beta}-\bigvee_{i \in I} \bigvee_{\beta>\alpha} G L_{i}^{\beta}
$$

converges to $G$. Hence by the property of lower semicontinuity of hyporadical collections, for $\alpha$ sufficiently large, this dominates an interval $E$ in $\Omega_{2}$. But this means that $E$ is not cut by any $L_{i}^{\alpha}$ for $\alpha$ large, contrary to hypothesis. 
Now for the general width two case. This lemma provides a substitute for Lemma 2.2, and thus once the Disjointness Conjecture is verified for these lattices, the arguments of Theorem 3.9 will apply.

Lemma 4.4. Let $\mathscr{L}$ be a width two lattice. Suppose that $\Omega$ is a hyporadical collection that does not contain any completely non-CD intervals. Then the subcollection $\Omega^{\prime}$ of indecomposable intervals in $\Omega$ is radical.

Proof. Each interval $E$ in $\Omega$ corresponds to an interval of the square. Because $\mu$ may not have full support, this interval may not be unique. However, it is contained in a unique minimal closed interval. So we may suppose that $E$ is an interval of the square contained in its minimal closed support $\bar{E}$. This set is bounded by two decreasing curves. Let $\Gamma$ be the intersection of these two curves minus the horizontal and vertical line segments of positive measure that it contains. Then $\bar{E} \backslash \Gamma$ decomposes into a countable disjoint union of connected noncomparable intervals $\left\{E_{1}, E_{2}, \ldots\right\}$. Moreover, each $E_{i}$ has interior or is a horizontal or vertical line segment. Because of the minimality of $\bar{E}$, it follows that each $E_{i}$ is indecomposable (into noncomparable intervals). Now $\Gamma$ is noncomparable with all the $E_{i}$ and is completely non-CD. From the fact that $\Omega$ is decomposable and $\Gamma$ is not in $\Omega$, it follows that some $E_{i}$ belongs to $\Omega$. Hence, as in Lemma 2.2, each interval $E$ in $\Omega$ may be replaced by this smaller indecomposable interval that it contains. Thus, it may be assumed that our intervals are indecomposable (and connected). We will work with this smaller hyporadical collection $\Omega_{1}$.

Let $\mu$ be a measure on the square so that $\mathscr{L}$ is isomorphic to $\mathscr{L}(\mu)$. Now consider the lattice $\mathscr{L}^{\prime}$ corresponding to the measure $\mu+m$, where $m$ is planar Lebesgue measure on the square. We may suppose that each interval $E$ is an interval of the square contained in its minimal closed support. Associate to each $E$ the interval $E^{\prime}$ with the same support in $\mathscr{L}^{\prime}$. That is, one takes the corresponding interval in $\mathscr{L}(\mu)$ and adds the interval in $\mathscr{L}(m)$ corresponding to the minimal closed support. Call this new collection $\Omega^{\prime}$.

The point is that if $L^{\prime}$ is a lattice projection in $\mathscr{L}^{\prime}$ which cuts an interval $E^{\prime}$ in $\Omega^{\prime}$, then the corresponding interval $L$ of $\mathscr{L}$ must cut $E$. For otherwise, $E^{\prime}$ is divided into two intervals $F_{1}$ and $F_{2}$ such that one, say $F_{1}$, has positive $m$ easure but $0 \mu$-measure. As $F_{1}$ is an interval with positive Lebesgue measure, it has interior. But then $\overline{F_{2}}$ is a closed interval supporting $E$ which is disjoint from $\operatorname{int}\left(F_{1}\right)$. This contradicts our construction of $E^{\prime}$.

It is evident that $\Omega^{\prime}$ satisfies the hypotheses of the previous lemma. Thus there is a finite subcollection of intervals $\mathscr{E}^{\prime}=\left\{E_{1}^{\prime}, \ldots, E_{n}^{\prime}\right\}$ in $\Omega^{\prime}$ that cannot be cut by any $k$ lattice projections. Let $\mathscr{E}$ be the corresponding collection of intervals in $\Omega_{1}$. By the previous paragraph, we obtain that $\mathscr{E}$ cannot be cut by any $k$ lattice projections either. Hence $\Omega_{1}$ is radical.

We now return to the problem of constructing a radical family of disjoint intervals. The following lemma is the crux of our planar argument.

Lemma 4.5. Let $\Omega=\left\{E_{i}\right\}$ be a finite collection of connected intervals of the unit square which are not atoms. If $\Omega$ cannot be cut by any five decreasing Borel sets, then there is a decreasing set $L_{0}$ such that one of the following occurs:

(i) $L_{0}$ is closed and contains some $E_{i}$, and whenever $\xi \in L_{0}$, then $U(\xi)$ contains an $E_{j}$. 
(ii) $L_{0}^{\perp}$ is closed and contains some $E_{i}$, and whenever $\xi \in L_{0}^{\perp}$, then $L(\xi)$ contains an $E_{j}$.

Proof. Let

$$
L_{1}=\left\{\xi \mid U(\xi) \supseteq E_{i} \text { for some } i\right\}
$$

and

$$
L_{2}=\left\{\xi \mid L(\xi) \supseteq E_{i} \text { for some } i\right\}^{\perp} .
$$

Clearly, $L_{1}$ and $L_{2}$ are decreasing sets. Since $\Omega$ is finite, $L_{1}$ is closed and $L_{2}$ is open. If we suppose that the conclusion of the lemma fails to hold, then neither $L_{1}$ nor $L_{2}^{\frac{1}{2}}$ dominates any $E_{i}$. Let $\Omega_{0}$ be the collection of those sets $E_{i}$ which are not cut by $L_{1}$ or $L_{2}$. Thus $\Omega_{0}$ cannot be cut by any three decreasing sets. Clearly, $P\left(\Omega_{0}\right)$ is dominated by $L_{2} \backslash L_{1}$.

If $L_{1}$ and $L_{2}^{\perp}$ overlap, then the conclusion of the lemma follows trivially. Thus, we may suppose $\operatorname{dist}\left(L_{1}, L_{2}^{\frac{1}{2}}\right)=\delta>0$.

We shall construct three decreasing lines which "zig-zag" between $L_{1}$ and $L_{2}$, and which will cut all the intervals in $\Omega_{0}$, contrary to supposition. Start by letting $\xi_{0}=\left(x_{0}, y_{0}\right)$ be the intersection point of the boundary of $L_{1}$ and the part of the boundary of the square on the path from $(0,0)$ to $(0,1)$ to $(1,1)$. Next let $x=\sup \left\{t \mid\left(t, y_{0}\right) \notin L_{2}\right\}$, and take $\xi_{1}=\left(x_{1}, y_{1}\right)$ to be the point $\left(x, y_{0}\right)$. Continue, taking $y$ now to be $\inf \left\{t \mid\left(x_{1}, t\right) \notin L_{1}\right\}$ and $\xi_{2}=\left(x_{2}, y_{2}\right)=\left(x_{1}, y\right)$. We proceed in this way constructing a zig-zag path until we obtain either $x_{i}=1$ or $y_{i}=0$. Observe that this process terminates after a finite number of steps because each step moves in the direction of increasing $x$ or decreasing $y$ by at least $\delta$. Then take $C=\bigvee_{i \text { odd }} L\left(\xi_{i}\right)$, and note that $C^{\perp}$ is contained in $\bigvee_{i \text { even }} U\left(\xi_{i}\right)$.

If $E \in \Omega_{0}$ is not cut by $L_{1}, L_{2}$ or $C$, then it belongs to either $C \backslash L_{1}$ or $L_{2} \backslash C$. These sets decompose into the disjoint connected components

$$
C \backslash L_{1}=\dot{\bigvee}_{i \text { odd }} L\left(\xi_{i}\right) \backslash L_{1} \text { and } L_{2} \backslash C \subseteq \bigvee_{i \text { even }} U\left(\xi_{i}\right) \backslash L_{2}^{\perp}
$$

By the connectedness of $E$, it lies in one of these sets. Without loss of generality, let us suppose that $E$ is a subset of $L\left(\xi_{1}\right) \backslash L_{1}$. From the definition of $L_{2}$, $E$ is not contained in $L(\xi)$ for any $\xi \leq \xi_{1}$ with $\xi \neq \xi_{1}$.

For $\eta>0$, set $\zeta=(\eta,-\eta)$ and define

$$
C_{\eta}^{\prime}=\bigvee_{i \text { odd }} L\left(\xi_{i}+\zeta\right) \text { and } C_{\eta}^{\prime \prime}=\bigvee_{i \text { odd }} L\left(\xi_{i}-\zeta\right)
$$

It will be shown that for $\eta$ sufficiently small, one of these sets cuts each $E$ not cut by $L_{1}, L_{2}$ or $C$. Indeed, with $E$ as above, $E$ is not contained in $L\left(\xi_{1}+\zeta\right)$ or $L\left(\xi_{1}-\zeta\right)$. If it is not cut by $L\left(\xi_{i}+\zeta\right)$ for some positive $\eta, E$ must be supported on the line segment $\left\{\left(x, y_{0}\right) \mid x_{0} \leq x \leq x_{1}\right\}$. Similarly, if it is not cut by $L\left(\xi_{1}-\zeta\right)$ for $\eta$ small, $E$ must be supported on the line segment $\left\{\left(x_{1}, y\right) \mid y_{2} \leq y \leq y_{1}\right\}$. These two options are incompatible, as that would have $E$ supported on the singleton $\left\{\left(x_{1}, y_{1}\right)\right\}$, and hence it would be an atom. We conclude that for $\eta$ small enough, one of these sets cuts $E$. This is true for each interval of this finite collection. Thus there is an $\eta$ sufficiently small to work for all.

Note that if horizontal or vertical line segments have zero $\mu$-measure, the proof above works with only four cuts. 
The next theorem uses the previous lemma to obtain disjoint intervals with a special geometric configuration, and thus verifies the Disjointness Conjecture for width two lattices.

Theorem 4.6. Let $F_{1}, \ldots, F_{n}$ be a set of connected intervals in the square which cannot be cut by any five decreasing Borel sets; and suppose that $\Omega$ is a finite set of intervals such that for each $i,\left\{E \in \Omega: E \subseteq F_{i}\right\}$ has the same property. Then there is a pairwise disjoint collection $E_{0}, \ldots, E_{k}$ of intervals from $\Omega$ and points $\xi_{1}, \ldots, \xi_{k}$ in the square such that either

$$
E_{0} \subseteq \bigcup_{i=1}^{k} L\left(\xi_{i}\right) \quad \text { and } \quad E_{i} \subseteq U\left(\xi_{i}\right) \quad \text { for } i=1, \ldots, k
$$

or else

$$
E_{0} \subseteq \bigcup_{i=1}^{k} U\left(\xi_{i}\right) \quad \text { and } \quad E_{i} \subseteq L\left(\xi_{i}\right) \quad \text { for } i=1, \ldots, k .
$$

In particular, $\left\{E_{0}, \ldots, E_{k}\right\}$ is a family of disjoint intervals which cannot be cut by a single lattice projection.

Proof. We apply the previous lemma to the intervals $F_{i}$. Suppose that we have obtained a closed decreasing $L_{0}$ which contains $F_{1}$, and such that $U(\xi)$ contains some $F_{i}$ whenever $\xi \in L_{0}$. For each $\xi$ in the boundary of $L_{0}, U(\xi)$ contains some $F_{i}$, and so contains a family of intervals of $\Omega$ which cannot be cut by any five decreasing sets.

Let $\Omega_{1}$ be the set of intervals in $\Omega$ which are contained in $F_{1}$. Now apply the lemma again, this time to the intervals of $\Omega_{1}$, to obtain a decreasing set $L_{1}$. If $L_{1}$ satisfies the second possible property allowed for in the lemma, then by choosing $\xi$ in $L_{0} \backslash L_{1}$ (which must be possible since this contains an interval of $\Omega_{1}$ ), both $L(\xi)$ and $U(\xi)$ will contain intervals and the result follows. We may thus suppose that $L_{1}$ satisfies the first alternative.

We now describe the fundamental construction. For any fixed $\xi_{0}$ in $L_{1}$, $U\left(\xi_{0}\right)$ contains an interval $G$ in $\Omega_{1}$. Now let $\xi_{0}^{\prime}$ be the smallest $\xi$ such that $L(\xi)$ contains this interval. If $U\left(\xi_{0}^{\prime}\right)$ contains an $E \in \Omega$ disjoint from $L_{0}$, then we are done. So we suppose that this never happens for any choice of $\xi_{0}$ and, in particular, it follows that $\xi_{0}^{\prime} \notin L_{0}$. Now, if $\xi_{0}^{\prime}=\left(x_{0}^{\prime}, y_{0}^{\prime}\right)$, let $\xi_{0}^{\prime \prime}=\left(x_{0}^{\prime \prime}, y_{0}^{\prime \prime}\right)$, where $x_{0}^{\prime \prime}=\sup \left\{t:\left(t, y_{0}^{\prime}\right) \in L_{0}\right\}$ and $y_{0}^{\prime \prime}=y_{0}^{\prime}$. Since $\xi_{0}^{\prime \prime} \in L_{0}, U\left(\xi_{0}^{\prime \prime}\right)$ contains a collection of intervals of $\Omega$ which are disjoint from $L_{0}$ and which cannot be cut by any decreasing set. Thus, in particular, these intervals are not all cut by the decreasing set $U\left(\xi_{0}^{\prime}\right)^{\perp}$ and, since we have supposed that $U\left(\xi_{0}^{\prime}\right)$ does not contain any of these intervals, there is an interval of $\Omega$ disjoint from $L_{0}$ which is contained in $U\left(\xi_{0}^{\prime \prime}\right) \backslash U\left(\xi_{0}^{\prime}\right)$. Since we started this construction with $\xi_{0}$, we shall name this interval finally obtained $E\left(\xi_{0}\right)$. Following this procedure for any $\xi \in L_{1}$, we obtain corresponding $E(\xi) \perp L_{0}$. Observe, of course, that $E(\xi) \subseteq U\left(\xi^{\prime \prime}\right) \subseteq U(\xi)$. Figure 1 below may be of some help.

The point of this construction is the following property which we claim for the $E(\xi)$. Given $\xi_{i}=\left(x_{i}, y_{i}\right)$ in the boundary of $L_{1}$ for $i=1,2$, let us suppose $\xi_{1} \ll \xi_{2}$, where this means $x_{1}<x_{2}$ and $y_{1}>y_{2}$. Then either $E\left(\xi_{1}\right)$ and $E\left(\xi_{2}\right)$ are disjoint or else $U\left(\xi_{1}\right)$ contains $E\left(\xi_{2}\right)$. The claim is justified by taking $\xi_{1} \ll \xi_{2}$ and supposing that $U\left(\xi_{1}\right)$ does not contain $E\left(\xi_{2}\right)$. Let $\xi_{i}^{\prime}=\left(x_{i}^{\prime}, y_{i}^{\prime}\right)$ and $\xi_{i}^{\prime \prime}=\left(x_{i}^{\prime \prime}, y_{i}^{\prime \prime}\right)$ be as in the construction above. We are 


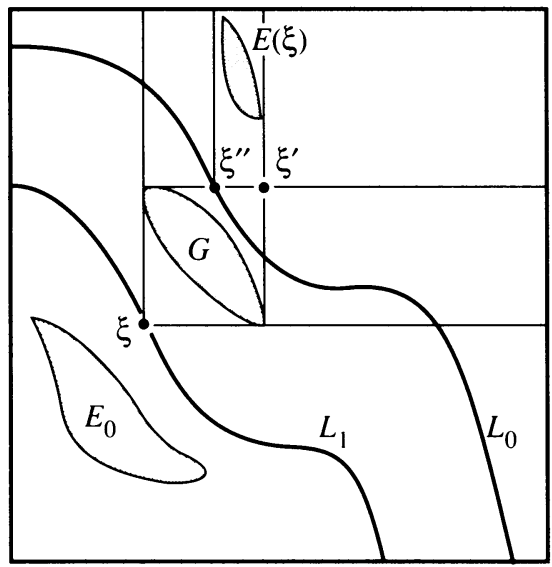

FIGURE 1

supposing that $\xi_{2}^{\prime \prime} \nsupseteq \xi_{1}$, so either $x_{2}^{\prime \prime}<x_{1}$ or $y_{2}^{\prime \prime}<y_{1}$. But $x_{2}^{\prime \prime} \geq x_{2} \geq x_{1}$, so we must have $y_{2}^{\prime \prime}<y_{1}$. Now consider $x_{1}^{\prime}$ and $x_{2}^{\prime \prime}$. If $x_{2}^{\prime \prime}$ were strictly less than $x_{1}^{\prime}$, then since $\left(x_{1}^{\prime}, y_{1}\right)$ belongs to the decreasing set $L_{0}$ (by the minimality of the choice of $\left.\xi_{1}^{\prime}\right)$, we would have

$$
x_{2}^{\prime \prime}<x_{1}^{\prime} \text { and } y_{2}^{\prime \prime}<y_{1} \text {. }
$$

This would contradict the assumption that $\xi_{2}^{\prime \prime}$ lies on the boundary of $L_{0}$. Thus we conclude that $x_{1}^{\prime} \leq x_{2}^{\prime \prime}$. But

$$
E\left(\xi_{1}\right) \subseteq\left\{(x, y) \mid x<x_{1}^{\prime}\right\} \text { and } E\left(\xi_{2}\right) \subseteq\left\{(x, y) \mid x \geq x_{2}^{\prime \prime}\right\}
$$

So $E\left(\xi_{1}\right)$ and $E\left(\xi_{2}\right)$ are disjoint.

Now, finally, we consider all possible pairwise disjoint collections of intervals $E(\xi)$ for $\xi$ on the boundary of $L_{1}$. Order such collections by taking one collection greater than another if every $\xi$ on the boundary of $L_{1}$ for which $U(\xi)$ contains an interval in the smaller collection also contains an interval in the larger. Select a maximal collection (which exists since $\Omega$ is finite). We claim that for each $\xi$ on the boundary of $L_{1}, U(\xi)$ contains an interval in this maximal collection. For otherwise, take $\xi_{0}$ for which this does not hold. Note that the set of points on the boundary of $L_{1}$ is «-totally ordered. Now, by the last paragraph, $E\left(\xi_{0}\right)$ is disjoint from all $E(\xi)$ in the maximal collection with $\xi_{0} \ll \xi$. On the other hand, if we delete from the collection all $E(\xi)$ which meet $E\left(\xi_{0}\right)$ and include $E\left(\xi_{0}\right)$, we have formed a pairwise disjoint collection which is larger (in our ordering), contradicting the assumptions of maximality.

That $\left\{E_{0}, \ldots, E_{k}\right\}$ cannot be cut by a single lattice projection is evident. For in order to cut each $E_{i}$ for $i \geq 1$, a projection $P$ must meet $U\left(\xi_{i}\right)$ and hence dominates $L\left(\xi_{i}\right)$ for all $i$. Hence $P$ dominates $E_{0}$. The second alternative is the same.

Corollary 4.7. The Disjointness Conjecture holds for all width two lattices with the constant $M=f(5,5)=35$.

Proof. This is an immediate consequence of the previous theorem and Lemma 2.4 .

Finally, we have achieved the main result of this paper. 
Theorem 4.8. Let $\mathscr{L}$ be a width two commutative subspace lattice. Then the Ringrose ideal is equal to the Jacobson radical of $\operatorname{Alg}(\mathscr{L})$.

Proof. The previous corollary verifies the Disjointness Conjecture for width two lattices. Also Lemma 4.4 shows that even when $\mathscr{L}$ is not completely distributive, hyporadical collections yield radical indecomposable collections. Thus the result now follows from Theorem 3.9.

Remark 4.9. The geometry of the square permitted the construction of a very special kind of bush. Namely, one of the two families $\Phi$ and $\Psi$ is always a singleton. Thus this has the structure of a tree, and iteration yields a tree of arbitrary length. The advantage of trees is that the interpolating operator can be written down explicitly. Precisely, if the first possibility of Theorem 4.6 occurs, choose subprojections $E_{0 i}$ of $E_{0}$ so that $E_{0 i} \leq L\left(\xi_{i}\right)$ and $E=\sum_{1 \leq i \leq n} E_{0 i}$. Then suppose that $E_{i} X \xi_{i}=\zeta_{i}$, where $1=\left\|\xi_{i}\right\| \leq\left\|\zeta_{i}\right\|$. Define

$$
\xi_{0 i}=\left\|E_{0 i} \xi_{0}\right\|^{-1} E_{0 i} \xi_{0} \text {. }
$$

Then set

Then

$$
A=\sum_{1 \leq i \leq n} \xi_{0 i} \otimes \zeta_{i}^{*}
$$

$$
A \sum_{1 \leq i \leq n}\left\|E_{0 i} \xi_{0}\right\| \zeta_{i}=\xi_{0}
$$

This makes the constructions of the previous section simpler.

\section{REFERENCES}

1. C. Apostol and K. R. Davidson, Isomorphisms modulo the compact operators of nest algebras. II, Duke Math. J. 56 (1988), 101-127.

2. W. B. Arveson, Operator algebras and invariant subspaces, Ann. of Math. (2) 100 (1974), 433-532.

3. K. R. Davidson, Nest algebras, Pitman Res. Notes in Math., vol. 191, Longman Sci. Tech., London and New York, 1988.

4. J. 20 (1990), 317-330.

5. K. R. Davidson and D. R. Pitts, Compactness and complete distributivity for commutative subspace lattices, J. London Math. Soc. (2) 42 (1990), 147-159.

6. A. Hopenwasser, The radical of a reflexive operator algebra, Pacific J. Math. 65 (1976), 375-392.

7. (1980), 124-126.

8. A. Hopenwasser and D. R. Larson, The carrier space of a reflexive operator algebra, Pacific J. Math. 81 (1979), 417-434.

9. J. R. Ringrose, On some algebras of operators, Proc. London Math. Soc. (3) 15 (1965), 61-83.

10. B. Wagner, Weak limits of projections and compactness of subspace lattices, Trans. Amer. Math. Soc. 304 (1987), 515-535.

Pure Mathematics Department, University of Waterloo, Waterloo, Ontario, Canada N2L 3G1

E-mail address: krdavids@math.waterloo.edu

Department of Mathematics, University of Nebraska, Lincoln, Nebraska 68588

E-mail address: jorr@anor.unl.edu 